\title{
L'apprentissage collaboratif : une forme de recherche collective reconfigurée par l'usage des TIC ?
}

\section{Collaborative learning: a type of collective research reconfigured by the use of ICT ?}

\author{
Alain Baudrit ${ }^{1 *}$ \\ ${ }^{1}$ Université de Bordeaux, Laboratoire LACES, 33076 Bordeaux Cedex, France.
}

\begin{abstract}
Résumé. L'apprentissage collaboratif est une forme d'activité collective dont on peut trouver les origines notamment chez Dewey [1] qui préconisait la pratique d'enquête (practical inquiry) au sein de l'institution scolaire. Confrontés à des situations incertaines ou problématiques, les élèves réunis en groupes peuvent ainsi explorer, expérimenter ou rechercher ensemble, ce qui peut les amener à faire des découvertes en matière de savoirs ou de connaissances. Réalisable en présentiel, ce type d'activité collective l'est également à distance grâce à l'usage des TIC (Technologies de l'Information et de la Communication). Est-ce que ces dernières sont de nature à reconfigurer une telle activité ? Si oui, dans quels sens ? Est-il possible d'envisager des formes d'hybridation (combinaison de dispositifs en présentiel et à distance) en termes d'apprentissages ou d'acquisitions de connaissances? Ces questions sont examinées sur la base de travaux réalisés dans les domaines de l'éducation et de la formation.
\end{abstract}

\begin{abstract}
Collaborative learning is a type of collective activity whose origins can be found in particular in Dewey [1] who advocated practical inquiry within the school institution. Confronted with uncertain or problematic situations, the pupils gathered in groups can thus explore, experiment or research together, which can lead them to make discoveries in terms of knowledge. This kind of collective activity can be carried out face-to-face and can also be done remotely with the use of ICT (Information and Communication Technologies). Are the latter likely to reconfigure such activity? If so, in what sense? Is it possible to envisage hybridization (combination of face-to-face and remote devices) in terms of learning or knowledge acquisitions? These questions are examined on the basis of researches carried out in the fields of education and training.
\end{abstract}

\section{Introduction}

Il n'existe pas à proprement parler de définition de l'apprentissage collaboratif (AC). Mais les auteurs qui se sont intéressés à cette forme d'apprentissage ont quand même essayé d'apporter des précisions à ce sujet, comme ils ont cherché à identifier des origines chez des auteurs ou dans des courants de pensée. Un nom revient souvent : John Dewey. L'influence de ce philosophe américain est perceptible à plus d'un titre. Nous lui devons l'idée de la pratique d'enquête (practical inquiry) qu'il considère comme « une transformation contrôlée ou dirigée d'une situation indéterminée en une situation déterminée dans ses distinctions et relations constitutives » [1] (p. 104-105; trad. pers.). En somme, il s'agit pour les apprenants de trouver du sens dans une situation qui, au départ, présente un caractère ambigu ou contradictoire. Mais il met également en avant le côté collectif de cette entreprise avec ce qu'il nomme la «communauté d'enquête » (community of inquiry) [2]. Ce type de communauté est en général constitué de personnes amenées à « résoudre de façon commune et conjointe une situation problématique » [3] (p. 262).

L'Ecole de Genève peut également être citée comme source d'influence avec son maître à penser : Jean Piaget. Celuici voit en effet dans l'autonomie des apprenants une condition indispensable à l'émergence de "conduites susceptibles d'éduquer l'esprit critique, l'objectivité et la réflexion discursive » [4] (p. 263). Dans le cadre de l'AC, les interlocuteurs sont donc amenés à interagir librement afin de les protéger de toute tentative d'influence externe susceptible de contrarier l'activité de recherche, de parasiter le fonctionnement de la pensée collective » [5] (p. 16-17). L'apport du psychologue

${ }^{*}$ Corresponding author: author@email.org 
russe Vygotski n'est pas non plus négligeable en tant qu'initiateur de la théorie socio-constructiviste. Ce faisant, il introduit la dimension sociale dans les apprentissages : «C'est essentiellement par le dialogue entre les membres du groupe et le partage de leurs savoirs mais aussi par la coordination de leurs actions qu'ils parviennent à résoudre un problème partagé et à construire de nouvelles connaissances " [3] (p. 262). Les interactions à caractère constructif sont donc valorisées en pareille circonstance, elles ont leur raison d'être particulièrement « lorsque les individus encouragent et facilitent les efforts de chacun pour atteindre le but du groupe » [6] (p. 173). Le but des groupes collaboratifs est bien de dénouer des situations ambigües où la participation de l'ensemble des partenaires de travail parait essentielle dans la recherche de solutions aux problèmes posés.

Telles sont les bases théoriques sur lesquelles repose l'AC. Ainsi, il peut être assimilé à un travail d'enquête mené de façon collective par un groupe de personnes qui, de façon autonome, gèrent leurs interactions dans le but d'appréhender et de surmonter des situations qui leur posent problème. Des opportunités d'apprentissage ou d'acquisition de connaissances se présentent alors à elles. C'est par exemple le cas lorsque, à l'école, des groupes d'élèves sont confrontés à des tâches qui présentent un caractère de nouveauté. Elles les obligent à essayer plusieurs solutions possibles, à échanger à propos de chacune d'elles, à confronter leurs points de vue respectifs, voire à expérimenter pour parvenir à une ou des solutions qui fassent consensus. Un exemple de ce type est présenté ci-dessous.

\section{L’apprentissage collaboratif en présentiel : une véritable activité collective ?}

L'exercice du « carré magique » (présenté ci-dessous) consiste à calculer des nombres avant de les placer dans une grille où l'un d'eux est déjà positionné. Il sert alors de point de repère pour le placement des autres nombres. Cette activité de recherche numérique a été proposée à 52 élèves de l'enseignement élémentaire, dont l'âge moyen est de 7,5 ans, associés en dyades [7]. Des épreuves initiales passées individuellement, toujours relatives à des activités de recherche numérique, font état de difficultés plus ou moins importantes rencontrées par ces élèves pour résoudre ce genre de situation-problème.

«Trouver l'emplacement de ces nombres dans la grille»

Verticalement :

$(1000 \times 2)+(100 \times 5)+3$

$(1000 \times 4)+(100 \times 7)+(10 \times 3)+1$

$(1000 \times 2)+(100 \times 4)+(10 \times 2)+4$

$(1000 \times 4)+(10 \times 5)+7$

Horizontalement :

$(1000 \times 4)+(100 \times 5)+7$

$(1000 \times 2)+(10 \times 5)+3$

$(1000 \times 2)+(100 \times 2)+(10 \times 4)+4$

$(1000 \times 4)+(100 \times 3)+(10 \times 7)+1$

\begin{tabular}{|l|l|l|l|}
\hline & $\mathbf{2}$ & & \\
\hline & $\mathbf{5}$ & & \\
\hline & $\mathbf{0}$ & & \\
\hline & $\mathbf{3}$ & & \\
\hline
\end{tabular}

Voici une séquence dyadique retranscrite où chaque intervention, action ou interaction fait l'objet d'un codage. Les deux élèves (Marina et Romain) s'y sont pris de la façon suivante pour réaliser l'activité demandée :

Lecture silencieuse de l'exercice à réaliser (1). Marina : "Tu comprends quelque chose toi ? " (2) ; Romain : "Non» (3). Il relit l'énoncé et explique à sa camarade: "Il faut placer les nombres verticalement et horizontalement » (4). Il accompagne ses commentaires de gestes verticaux et horizontaux (5). Marina : "Par exemple, là ça fait 4507 » (6) ; Romain : "Ça fait plutôt 4057 » (hésitation) (7). Il s'aperçoit de son erreur et place 4507 dans la grille (8). Marina essaie de trouver le nombre suivant (2 053) (10); pendant ce temps, Romain place le troisième nombre (2 244) (11). Marina fait observer une inversion entre les cases horizontales et verticales de la grille (12). Elle efface les nombres écrits jusqu'à présent (13). Romain : "Je n'y comprends rien du tout » (14). Marina : "La verticale, c'est comme ça » (Gestes à l'appui) (15). Romain écrit de nouveau (16). Marina: "Tu sais ce que tu fais?" (17) ; Romain : "Oui, il me semble " (18). Elle lui explique son erreur (placement horizontal des nombres verticaux) (19). Elle prend la fiche et efface: "On va recommencer" (20). Romain : "Ah oui! Il faut mettre 4507 comme ça (horizontalement) » (21) ; Marina : "Oui, c'est ce qu'on va faire » (22). Ils placent le nombre 4507 ; l'un écrit (Marina), l'autre vérifie (Romain) (23). [7] (p. 121-122).

Cette séquence a été analysée de la façon suivante : «La première partie de cette séquence est composée de recherches collectives (3-4-5-6-7-9) et d'activités plus individuelles (8-10-11). À partir de l'interaction 12, les échanges évoluent. Marina s'aperçoit de l'erreur initiale (les nombres verticaux sont placés horizontalement). Romain la réitère immédiatement après (15-16-17). Sa camarade l'aide à comprendre le placement des nombres (19-20). La stratégie la plus sûre (commencer par les nombres horizontaux) est ensuite découverte collectivement (21-22). Ils la mettent en œuvre grâce à une complémentarité de rôles (23). Les échanges ne se limitent pas aux interactions verbales, la communication non verbale est également présente (5-15) » [7] (p. 122). Les principaux ingrédients de l'AC sont repérables à l'intérieur 
de cette séquence : travail autonome, confrontation à une situation problématique, explorations par essais/erreurs, large registre interactif (communication verbale et non verbale). De plus, il est à noter quelques phases (7-11-17) où les partenaires de travail font état de divergences qui, tout naturellement, sont suivies de tentatives d'accord. Le processus de réparation est reconnaissable ici, processus ainsi décrit : «La nécessité de parler de sujets qui ne font pas l'objet de significations partagées n'est pas un problème mais, en fait, un avantage (...). Afin de démêler ces différences d'interprétations, les participants sont obligés d'expliciter leurs présupposés, d'argumenter, de raisonner et de fournir des exemples » [8] (p. 42 ; trad. pers.). Il devient possible de parler de collaboration contradictoire à partir du moment où il y a une sorte de " choc des idées qui suscite le besoin de réexaminer, revoir, et justifier sa propre vision du monde » [9] (p. 143 ; trad. pers.). À cette occasion, comment ne pas évoquer non plus la notion de conflit socio-cognitif, chère aux néo-piagétiens de l'École de Genève [10] [11], qui voient là des interactions sociales à même d'obliger «le sujet à coordonner ses actions avec celles d'autrui (et) l'entraînent dans un processus de décentration qui l'engage dans un conflit entre son point de vue et celui de ses partenaires » [10] (p. 136). L'AC ne saurait donc se résumer à un seul mode d'échanges, il est susceptible de prendre différentes configurations en fonction des situations-problèmes à résoudre, des caractéristiques des partenaires de travail en présence ou des modes de communication dont ils disposent. Sur ce dernier point d'ailleurs, il convient de voir comment les TIC (Technologies de l'Information et de la Communication) sont de nature à jouer un rôle en matière d'AC, si elles modifient fondamentalement les échanges entre les personnes concernées ou le registre interactif des partenaires de travail qui maintenant communiquent à distance.

\section{L'apprentissage collaboratif à distance : de nouvelles façons d'interagir ?}

Une étude réalisée à l'Université de Taïwan est de nature à apporter quelques éclaircissements sur cette question [12]. Elle a été menée auprès de 52 étudiants qui suivent des cours dans différents domaines scientifiques dans le but de se préparer aux métiers de l'enseignement. Il est surtout question qu'ils acquièrent une posture scientifique afin que leurs futurs élèves soient sensibilisés à la même démarche. Pour ce faire, ils ont la possibilité d'interagir par le biais du forum KF (Knowledge Forum) dans le but de «mener des enquêtes scientifiques et de construire des concepts scientifiques grâce à la collaboration en ligne » [12] (p. 2 ; trad. pers.). S'agissant de cours d'introduction aux sciences, les enquêtes en question portent en général sur des problèmes liés à la vie quotidienne comme par exemple : «Pourquoi les sacs en plastique ne se décomposent-ils pas ? ». Ce genre de situation présente l'avantage d'amener les étudiants à « construire des connaissances à partir de problèmes authentiques, d'idées réelles qui montrent que les connaissances résultent des efforts produits par les apprenants dans la compréhension du monde qui les entoure » [12] (p. 7 ; trad. pers.). Dans cette optique également, les étudiants ne sont pas contraints par des consignes particulières en matière d'investigation scientifique et, ce faisant, ils ont la possibilité d'appréhender un fait ou un problème à leur manière. En ce sens, leurs enquêtes présentent un caractère semi-structuré, elles sont axées « sur les étudiants mêmes qui, à mesure qu'ils identifient les problèmes à résoudre, réexaminent et adaptent leurs plans d'enquête en conséquence [13] [14]» [12] (p. 5 ; trad. pers.). Enfin, à l'image des scientifiques qui travaillent de manière collective, les étudiants concernés sont invités à faire de même à l'appui du forum KF qui « offre plusieurs façons d'engager un travail collaboratif sur les idées. Les participants peuvent s'appuyer sur ou annoter les messages des autres, co-rédiger des notes, ajouter des mots-clés, et créer des notes de type «rise-above » ou « résumés » qui intègrent les idées associées aux problèmes sur lesquels la communauté enquête » [12] (p. 7 ; trad. pers.). C'est dans ce cadre expérimental que les auteurs de cette étude essaient de répondre à la question suivante : «Quels types d'activités en ligne contribuent le plus à améliorer la qualité des enquêtes scientifiques menées par les étudiants ?» [12] (p. 6 ; trad. pers.).

Des études antérieures ont déjà permis de dégager quelques enseignements sur les mécanismes interactifs à l'œuvre lorsqu'il s'agit de se livrer à des constructions théoriques dans le but d'accéder à de véritables concepts scientifiques. Il est notamment établi que «pour engager un travail innovant de construction des connaissances, les membres des communautés n'ont pas seulement besoin de produire des idées (contribuer) et de prendre connaissance des idées des autres (lire), ils doivent également étendre leur raisonnement et échanger des idées (collaborer), construire, composer et examiner l'ensemble des idées (progresser) [15]» [12] (p. 9 ; trad. pers.). Dans la continuité de ces observations, les auteurs de cette étude ont examiné les échanges enregistrés sur le forum KF au niveau de quatre points : «L'activité contributive, indexée sur le nombre moyen de notes postées par chaque étudiant, l'activité de lecture, indexée sur le nombre de fois qu'une note est lue, l'activité collaborative, indexée sur le nombre d'échanges suscités par une note, et l'activité d'approfondissement, indexée sur le nombre de fois qu'une note est réexaminée et le nombre de supports utilisés pour l'investigation scientifique » [12] (p. 9 ; trad. pers.). Les nombres indiqués entre parenthèses dans le schéma présenté ci-dessous représentent ces quatre valeurs pour chaque note postée par les étudiants. 


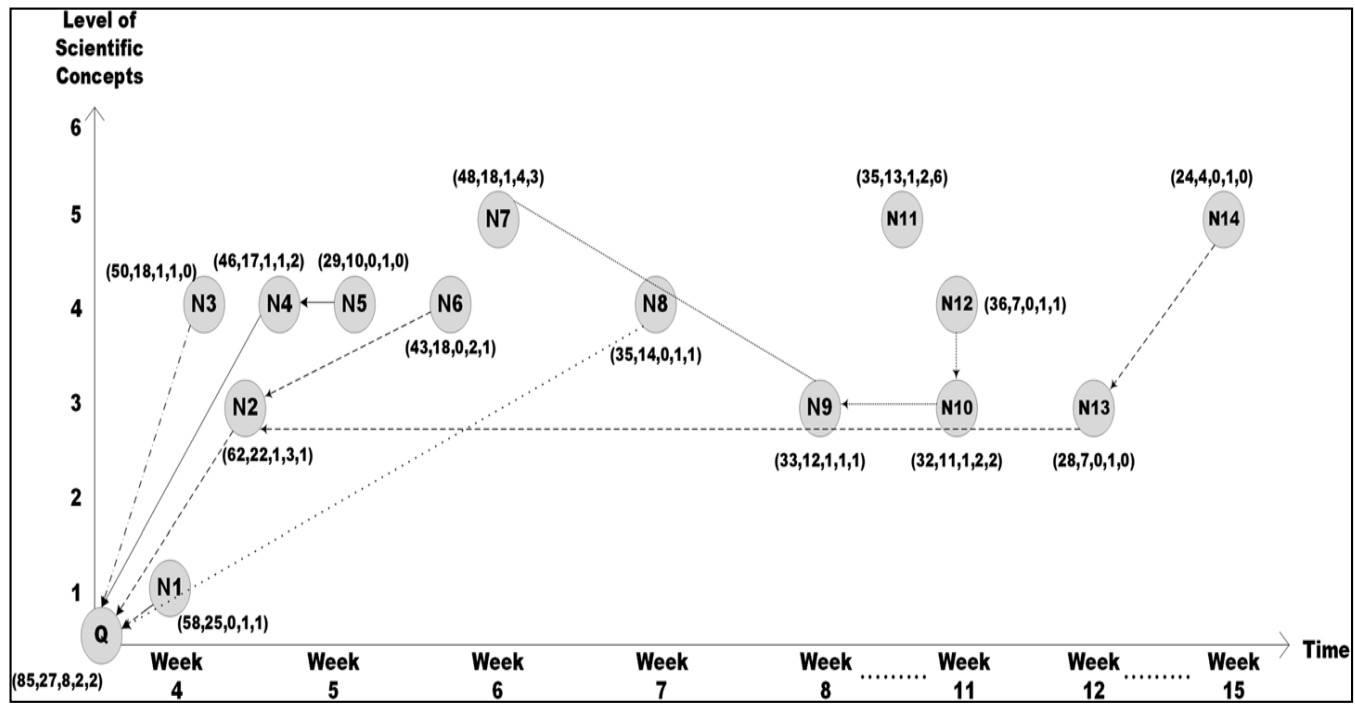

Figure 1 : Chronologie des notes postées sur le forum KF [12] (p. 14).

C'est ainsi que les auteurs analysent une séquence interactive relative à l'étude de l'effet Coriolis initiée par une question posée par un étudiant : «Je voudrais savoir pourquoi l'eau tourne dans lavabo ?». Toute une série d'échanges va alors s'ensuivre.

Le premier est ainsi formulé : «C'est parce que tu pousses l'eau lorsque tu te laves les mains ». Une réponse différente est ensuite apportée par une autre étudiante : « C'est à cause du champ magnétique, la direction du tourbillon est différente dans les hémisphères sud et nord ». Les auteurs font alors l'observation suivante : «Cette étudiante a introduit le concept de champs magnétiques ... néanmoins elle n'a pas précisé comment les champs magnétiques fonctionnent ou comment ils pourraient influencer le sens d'écoulement de l'eau » [12] (p. 14 ; trad. pers.). Deux explications alternatives sont ensuite proposées par une autre étudiante : «Je pense que c'est en raison de la forme ronde du lavabo. Lorsque l'eau coule vers le centre, les molécules se serrent les unes contre les autres formant ainsi un tourbillon pour que l'eau pénètre dans le siphon ... Une autre idée est l'effet Coriolis. L'eau s'écoule vers le siphon de façon courbe ». Cette dernière interprétation a incité l'intervenante précédente à rectifier son point de vue basé sur l'action des champs magnétiques : «Étant donné que la direction de l'écoulement de l'eau est différente dans les hémisphères nord et sud, je reconnais que l'idée de l'effet Coriolis paraît plus plausible. C'est parce que la Terre tourne ... L'idée initiale des champs magnétiques que j'ai intuitivement suggérée a peu à voir avec le phénomène ». L'enseignant du cours pose alors une question : «Qu'est-ce que l'effet Coriolis ? », voici la réponse d'un étudiant : « Nous avons découvert l'effet Coriolis au lycée. Il est causé par la rotation de la Terre ». Pour finir, un étudiant résume la situation de la façon suivante : « Nous pensons que c'est dû à l'effet Coriolis, mais dans l'espace réduit du lavabo d'autres facteurs peuvent également influencer l'écoulement de l'eau comme la surface de frottement, la viscosité, l'endroit du siphon, la forme du lavabo » [12] (p. 1415 ; trad. pers.). Que dire de cette série d'échanges initiée par une observation liée à la vie quotidienne, à savoir la rotation de l'eau dans un lavabo ?

Les auteurs de cette étude notent que cette observation formulée sur le mode interrogatif a bien suscité des échanges. Elle n'a pas laissé les étudiants indifférents avec des niveaux de réponse plutôt hétérogènes : «Tu pousses l'eau lorsque tu te laves les mains »; «Les molécules se serrent les unes contre les autres formant ainsi un tourbillon »; «La direction de l'écoulement de l'eau est différente dans les hémisphères nord et sud »... Une évolution est toutefois perceptible en ce que l'effet Coriolis est finalement évoqué parce qu'il a fait l'objet d'un enseignement auparavant. Est-il bien maîtrisé par les étudiants ? Quelle connaissance en ont-ils véritablement ? Ces questions restent en suspens mais les auteurs font quand même cette observation : « Lire les messages des autres n'a pas amélioré la qualité des idées des étudiants. Ce n'est que lorsque les étudiants ont soumis leurs idées au groupe, connecté leurs idées à celles des autres, et continuellement révisé leurs idées au cours de l'activité en ligne qu'ils sont parvenus à une meilleure compréhension des concepts scientifiques » [12] (p. 16 ; trad. pers.). Leur niveau de compréhension reste peut-être encore imparfait mais, à force de retouches successives à la faveur d'échanges à distance, ils ont franchi quelques paliers qui leur ont permis de s'affranchir d'interprétations non scientifiques du phénomène étudié. Parallèlement, l'accès à des connaissances plus affirmées se trouve facilité.

La question initiale posée par les auteurs de cette étude est la suivante : «Quels types d'activités en ligne contribuent le plus à améliorer la qualité des enquêtes scientifiques menées par les étudiants ? » [12] (p. 6 ; trad. pers.). Lorsqu'ils se livrent à un examen critique de leurs idées ou interventions respectives, ils semblent bien se donner les moyens de progresser en matière de connaissances, d'aller chercher des interprétations plus scientifiquement fondées. Ce qui traduit finalement un investissement assez poussé dans l'activité collaborative, contrairement à des partenaires de travail qui se 
contenteraient de poster des idées sur un forum et d'en prendre simplement connaissance. Ce genre de travail réflexif paraît avoir partie liée avec la critique-constructive [16] dans laquelle il y a lieu de voir un regard analytique et attentif porté sur les idées et les faits. Voilà une démarche qui en général favorise les activités de questionnement dont l'importance est soulignée s'agissant de dynamiser l'AC [17] [18]. La qualité de la recherche collaborative menée par les étudiants paraît donc tenir à leur degré d'implication dans l'activité collective, notamment repérable dans l'exercice de la critique-constructive, elle-même source de questionnements susceptibles de stimuler les échanges en ligne. C'est de la sorte que la collaboration à distance semble autoriser des progrès en termes d'acquisition de connaissances chez des partenaires de travail éloignés les uns des autres.

\section{Discussion finale}

Collaborer en présentiel/collaborer à distance, voilà deux cas de figure significatifs d'une « activité collective déployée par des partenaires confrontés à une situation à fort degré d'incertitude » [19] (p. 112). Deux cas plutôt différentiables en matière d'interactions sociales, d'échanges mis en œuvre afin d'aborder et de tenter d'élucider ce genre de situation. Alors que dire de ces deux types d'AC? En quoi se distinguent-ils véritablement?

La collaboration en présentiel présente un caractère plutôt multiforme avec des interactions composites comme des occurrences d'entraides, d'oppositions, de coopérations ; tout ceci de façon plutôt irrégulière et informelle. Comme si les partenaires de travail utilisaient, de façon plus ou moins aléatoire, un maximum de moyens pour essayer de trouver des solutions aux problèmes qu'ils ont à résoudre. Ils ne cherchent pas à adopter une méthode de travail particulière, à suivre une démarche d'investigation préétablie ; ils agissent plutôt au coup par coup ou procèdent par essais/erreurs. Lorsqu'une tentative de résolution collective échoue, ils en essaient une autre jusqu'à ce qu'ils parviennent à leurs fins. Cette façon de faire est par exemple typique de la méthode GI (Group Investigation) [20], utilisée dans le système éducatif israélien, où les élèves « ont le libre choix de leurs partenaires, de leurs thèmes d'étude, de leurs façons de s'organiser, de leurs stratégies de travail (...). Tout est fait pour que des formes d'interdépendance assez souples soient adoptées par les pairs. La contrainte externe est réduite à sa plus simple expression » [21] (p. 57). De la sorte, la collaboration en présentiel paraît prendre les traits d'une exploration collective à caractère spontané.

De son côté, la collaboration à distance semble s'apparenter à une sorte de co-construction scandée par des retouches successives. Le processus de co-construction est ici entendu dans son acception classique, c'est-à-dire identifiable lors « de tâches mutuelles qui amènent les partenaires à travailler ensemble pour produire quelque chose qu'aucun ne peut produire seul »[22] (p. 329 ; trad. pers.). Deux ou plusieurs personnes sont ainsi associées pour mener à bien une activité qui, réalisée de manière individuelle, leur aurait posé bien plus de problèmes. Et lorsqu'une d'entre elles fait une proposition ou émet une idée, celle-ci est soumise à l'examen des partenaires de travail et fait l'objet de questionnements afin d'éprouver sa validité au regard du problème à résoudre. À partir de là une proposition ou une idée alternative, censée être plus recevable, peut venir d'un autre partenaire, elle est de la même façon passée au crible de la critique collective. Et ainsi de suite. Les participants progressent de la sorte, par étapes successives, assimilables à des paliers franchis un à un de manière évolutive. Ce qui leur permet de se dégager de savoirs plus ou moins approximatifs pour se rapprocher de connaissances plus scientifiquement fondées ou de concepts plus élaborés. Ainsi, la collaboration à distance pourrait bien se donner à voir sous la forme d'une co-construction à caractère graduel.

L'apprentissage collaboratif paraît bien être sérieusement reconfiguré par l'usage des TIC. Le caractère largement exploratoire de la collaboration en présentiel prend une forme plus structurée dans le cadre de collaboration à distance. Le côté spontané de la première, contraste avec la pente rationnelle prise par la seconde. En termes d'apprentissage, quand l'une permet des avancées par à-coups, l'autre autorise des progrès inscrits dans la régularité. Les interactions multiformes observées dans un cas deviennent plus homogènes dans l'autre. Voilà des différences ou des distinctions qui rendent ces deux types d'AC peu compatibles, peu propices à d'éventuelles associations. Ceci à l'heure où, pourtant, les dispositifs hybrides deviennent de plus en plus courants et conseillés parce que propices à la constitution d'un "mélange équilibré et harmonieux de la présence et de la distance soutenue par l'usage des technologies » [23] (p. 17). S'agissant d'apprentissage collaboratif, que dire de telles tentatives d'hybridation? N'est-il pas risqué de faire interagir des partenaires de travail à la fois en présentiel et à distance tellement les deux activités sont contrastées ? Brouillage et parasitage des échanges ne sont-ils pas possibles en pareil cas ? À l'évidence, une telle cohabitation suppose quelques liens ou relations de dépendance entre les deux situations interactives et, en ce sens, l'hypothèse de la complémentarité mériterait très certainement d'être mise à l'épreuve. Voilà une piste de recherche susceptible de fournir quelques éclaircissements sur d'éventuelles compensations réciproques entre leurs points forts et leurs points faibles respectifs. Affaire à suivre.

\section{References}

1. J. Dewey, The theory of inquiry. New York: Henry Holt and Company (1938).

2. J. Dewey, Démocratie et Éducation. Paris : Armand Colin (1916/1990).

3. A. Jézégou, Créer de la présence à distance en e-learning. Cadre théorique, définition, et dimensions clés. Distances \& Savoirs, 8, 2, 257-274 (2010). 
4. J. Piaget, Psychologie et Pédagogie. Paris : Denoël (1969).

5. A. Baudrit, L'apprentissage collaboratif: plus qu'une méthode collective? Bruxelles: De Boeck Supérieur (2007).

6. C. Buchs, L. Filisetti, F. Butera, A. Quiamzade, Comment l'enseignant peut-il organiser le travail de groupe ? In E. Gentaz \& P. Dessus (eds.), Comprendre les apprentissages. Sciences cognitives et éducation. Paris : Dunod, 169183 (2004).

7. A. Baudrit, Apprendre à deux. Etudes psychosociales de situations dyadiques. Paris : PUF (1997).

8. M. J. Baker, T. Hansen, R. Joiner, D. Traum, The role of grounding in collaborative learning. In P. Dillenbourg (ed.), Collaborative learning : Cognitive and computational approaches. Oxford : Pergamon, 31-63 (1999).

9. W. Damon, E. Phelps, Strategic uses of peer learning in children's education. In T. J. Berndt \& G. W. Ladd (eds.), Peer relationships in child development. New York : John Wiley \& Sons, 135-157 (1989).

10. A.-N. Perret-Clermont, La construction de l'intelligence dans l'interaction sociale. Berne : Peter Lang (1979).

11. W. Doise, G. Mugny, Le développement social de l'intelligence. Paris : Interéditions (1981).

12. P.-J. Li, H.-Y. Hong, C.-S. Chai, P.-Y. Lin, Fostering students' scientific inquiry through computer-supported collaborative knowledge building. Research in Science Education (2018). https://doi.org/10.1007/s11165-0189762-3

13. R. L. Bell, L. Smetana, I. Binns, Simplifying inquiry instruction. The Science Teacher, 72, 7, 30-33 (2005).

14. F. Riga, M. Winterbottom, E. Harris, L. Newby, Inquiry-based science education. In K.S. Taber \& B. B. Akpan (eds.), Science education: An international course companion. Rotterdam : Sense Publishers, 247-261 (2017).

15. H.-Y. Hong, F. R. Sullivan, Towards an idea-centered, principle-based design approach to support learning as knowledge creation. Educational Technology Research \& Development, 57, 5, 613-627 (2009).

16. J.-M. De Ketele, Préface. In M. Vial et N. Caparros-Mencacci (eds.), L'accompagnement professionnel ? Méthode à l'usage des praticiens exerçant une fonction éducative. Bruxelles: De Boeck Supérieur, 5-8 (2007).

17. M. Pedaste, M. Mäeots, L. A. Siiman, T. de Jong, S. A. N. van Riesen, E. T. Kamp, C. C. Manoli, Z. C. Zacharia, E. Tsourlidaki, Phases of inquiry-based learning: Definitions and the inquiry cycle. Educational Research Review, 14, 47-61 (2015).

18. Y. Tai, Y.-L. Ting, T.-H. Tseng, A proposed cohesive use of online discussion board from the aspects of instructional and social interactions in engineering education. International Journal of Online pedagogy and Course Design, $\mathbf{8}$, 3, 33-44 (2018). DOI:10.4018/IJOPCD.2018070103

19. A. Baudrit, Apprentissage collaboratif : des conceptions très éloignées des deux côtés de l'Atlantique ? Carrefours de l'éducation, 27, 103-116 (2009).

20. Y. Sharan, S. Sharan, Group investigation: Expanding cooperative learning. New York : Teacher's College Press (1992).

21. Baudrit A., L'apprentissage coopératif : origines et évolutions d'une méthode pédagogique. Bruxelles : De Boeck Supérieur (2005).

22. E. A. Forman, C. B. Cazden, Exploring vygotskian perspectives in education: The cognitive value of peer interaction. In J. Wertsch (ed.), Culture, communication and cognition. Cambridge: Cambridge University Press, 323-347 (1985).

23. D. Peraya, B. Charlier, N. Deschryver, Une première approche de l'hybridation. Étudier les dispositifs hybrides de formation. Pourquoi ? Comment? Éducation \& Formation, e-301, 15-34 (2014). 